\title{
Systematic characterization of somatic mutation-mediated microRNA regulatory network perturbations
}

Xu Hua ${ }^{1 \dagger}$, Yongsheng $\mathrm{Li}^{2 \dagger}$, Li Guo ${ }^{1 \dagger}$, Min Xu ${ }^{3}$, Dan $\mathrm{Qi}^{3}$, Jason H. Huang ${ }^{3,4}$, Akshay Bhat $^{2,5,6}$, Yunyun Zhou ${ }^{7}$, Erxi Wu ${ }^{2,3,4,8}$, and S. Stephen $\mathrm{Yi}^{2,5,6,9 *}$

${ }^{1}$ Department of Systems Biology, The University of Texas MD Anderson Cancer Center, Houston, TX 77030, USA

${ }^{2}$ Department of Oncology, LIVESTRONG Cancer Institutes, Dell Medical School, The University of Texas at Austin, Austin, TX 78712, USA

${ }^{3}$ Neuroscience Institute and Department of Neurosurgery, Baylor Scott \& White Health, Temple, TX 76502, USA

${ }^{4}$ Department of Surgery, Texas A \& M University Health Science Center, College of Medicine, Temple, TX 76508, USA

${ }^{5}$ Institute for Cellular and Molecular Biology (ICMB), College of Natural Sciences, The University of Texas at Austin, Austin, TX 78712, USA

${ }^{6}$ Oden Institute for Computational Engineering and Sciences (ICES), The University of Texas at Austin, Austin, TX 78712, USA

${ }^{7}$ Department of Data Science, University of Mississippi Medical Center, Jackson, MS 39216, USA

${ }^{8}$ Department of Pharmaceutical Sciences, Texas A \& M University Health Science Center, College of Pharmacy, College Station, TX 77843, USA

${ }^{9}$ Department of Biomedical Engineering, Cockrell School of Engineering, The University of Texas at Austin, Austin, TX 78712, USA

${ }^{\dagger}$ These authors contributed equally to this work.

${ }^{*}$ Correspondence: stephen.yi@austin.utexas.edu (S.Y.) 


\section{Abstract}

Somatic mutations are a major source of cancer development. Many driver mutations have been identified in protein coding regions. However, the function of mutations located in microRNAs (miRNAs) and their target binding sites along the human genome remains largely unknown. Here, we built comprehensive cancer-specific miRNA regulatory networks across 30 cancer types to systematically analyze the effect of mutations on miRNA related pathways. 3,518,261 mutations from 9,819 samples were mapped to miRNA-gene interactions (mGl), and mutations in miRNAs versus in their target genes show a mutually exclusive pattern in almost all cancer types. Using a linear regression method, we further identified 89 driver mutations in 14 cancer types that can significantly perturb miRNA regulatory networks. We find that driver mutations play their roles by altering RNA binding energy and the expression of target genes. Finally, we demonstrate that mutated driver gene targets are significantly down-regulated in cancer and function as tumor suppressors during cancer progression, suggesting potential miRNA candidates with significant clinical implications. We provide this data resource (CanVar-mGl) through a user-friendly, open-access web portal. Together, our results will facilitate novel non-coding biomarker identification and therapeutic drug design.

\section{Introduction}

Genetic changes are a primary source of oncogenesis. Somatic mutations accumulate in cells during one's lifetime. Among them, some may affect a gene's function or a regulatory element and lead to a phenotypic consequence, and are therefore often referred to as "driver mutations". Other variants may not have either phenotypic consequences or biological effects that are selectively advantageous to cancer cells, and are thus defined as "passenger mutations" (1). Many studies have paid attention to missense mutations in protein coding regions that can drive cancer development. A number of driver mutations have been identified in previous research efforts, such as BRAF (V600E), IDH1 (R132H), PIK3CA (E545K), EGFR (L858R), and KRAS (G12D) (2). However, in addition to missense mutations that affect the protein coding components of human genome, microRNAs (miRNAs) and their target binding sites 
occupy a significant proportion of the genome and can harbor somatic mutations which play driver roles through miRNA related pathways.

miRNAs are endogenous regulatory non-coding RNAs that are $\sim 22 \mathrm{nt}$ in length and act by targeting messenger RNAs (mRNAs) for cleavage or translational repression. The diversity and abundance of miRNA targets contribute to the complexity of gene regulatory networks. Increasing lines of evidence have demonstrated that miRNAs play critical functions in various developmental, physiological and pathological processes including cancer. Deregulation in the expression of miRNAs and their targets has been observed in various types of human cancer, such as glioma, breast cancer and prostate cancer. Mutations in miRNAs or their target genes may exert important effects on their deregulated expression. A number of studies have suggested that somatic mutations could impact miRNA-gene interactions and are related to cancer development $(3,4)$.

Several initial studies explored the mutational effect on miRNAs and their targets. SomamiR $(5,6)$ mapped mutations to miRNAs and their targets, and developed a tool to calculate enrichment of mutated targets on KEGG pathways. PolymiRTS (7-9) used a TargetScan score to evaluate the effects changed by mutations on miRNA targets. However, mutations in specific cancer types and in individual patients were not considered in these studies. Furthermore, the mechanism of how these mutations influence miRNA target genes was not clear. A study by Stegeman et al. used miRNA mimics and reporter gene assays and showed that mutations could change the expression of target genes in prostate cancer (10). But it remains elusive the extent to which mutations could impair miRNA-gene interactions, and a global view of driver mutation-mediated gene regulatory network perturbations on a pan-cancer scale is needed.

Recently, with the development of high throughput sequencing projects, such as TCGA and ICGC, numerous somatic mutations have been identified in various cancer types. These mutations might create new miRNA binding sites or lose several binding sites, which further perturb miRNA-gene regulation. To address these goals, we interrogated 
to what extent and how somatic mutations perturb miRNA-gene regulatory networks in cancer. In this study, we derived elaborate miRNA-target interaction networks in each cancer type by integrating empirically validated binding sites by CLIP-Seq, and expression correlation between miRNAs and target genes predicted by TargetScan (11). We demonstrate that somatic mutations are likely to occur selectively in miRNAs or target genes to perturb cancer hallmark-related functions. A mutually exclusive pattern is found for mutations in miRNAs and their targets. Driver mutations that significantly perturb miRNA regulatory networks in cancer are further identified. We show that driver mutations could exert their functions by impairing RNA binding affinity, resulting in alteration of target gene expression profiles. Intriguingly, driver gene targets that are significantly down-regulated in cancer often function as tumor suppressors during cancer progression. Our study provides a valuable resource for systematic investigation of the functional impact of somatic mutations on miRNA regulation in cancer.

\section{Materials and Methods}

\section{Identification of global miRNA-gene interactions (mGls)}

Argonaute (AGO) proteins are RNA-binding proteins (RBP) and essential components of the RNA-induced silencing complex (RISC) which is the molecular machinery for miRNA-induced silencing. High-throughput sequencing of immunoprecipitated RNAs after cross-link (CLIP-Seq) to AGO proteins provides powerful ways to trace the footprints of miRNA binding sites (12). We collected 36 AGO CLIP-Seq datasets from starBase v2.0 (13) and considered these binding sites as evidences of physical interactions between miRNAs and target genes. All the peaks of AGO CLIP-Seq data were merged together based on their genomic locations to obtain a global pool of miRNA-gene physical interactions. Peaks that were overlapped with at least $1 \mathrm{bp}$ were merged together and 434701 merged peaks were finally obtained after converting to hg38 genome assembly.

Although the AGO CLIP-Seq peaks could capture the footprints of miRNA binding sites, which miRNAs could bind to specific target sites still remains unclear. Agarwal, et al. showed that many non-canonical sites detected by cross-linking method do not mediate repression despite binding the miRNAs while the vast majority of functional sites are 
canonical and can be identified by TargetScan (v7.0) (11). In this study, to identify mGls and functional binding sites on the mRNA, 16,347,639 interactions between all the miRNAs in miRBase release 21 and target genes in whole human genome were downloaded from TargetScan (v7.0) (11). miRNA family name was mapped to mature miRNA name to get the miRNA-gene pair. Binding sites of each miRNA-gene pair were further intersected with AGO merged peaks and we finally obtained 279,924 miRNAgene interactions (mGls) among 2586 mature miRNAs and 4198 target genes as global reference.

\section{Identification of cancer-type specific miRNA-gene interactions (mGls)}

To obtain the functional mGls in specific cancer type, we considered the expression correlation among samples for each $\mathrm{mGl}$ of global reference in the cancer type. Gene expression quantification by RNA-Seq data and isoform expression quantification of mature miRNAs by miRNA-Seq data of 33 cancer types were downloaded from The Cancer Genome Atlas Project (TCGA). For each cancer type, datasets with more than 50 common samples (Table S1) between RNA-Seq and miRNA-Seq data were extracted and spearman's rank correlation of expression for each pair of miRNA and target gene was calculated.

$$
\rho_{i}=\frac{\sum_{k}^{N}\left(r m_{i k}-\overline{r m_{i}}\right)\left(r g_{i k}-\overline{r g_{i}}\right)}{\sqrt{\sum_{k}^{N}\left(r m_{i k}-\overline{r m_{i}}\right)^{2} \sum_{k}^{N}\left(r g_{i k}-\overline{r g_{i}}\right)^{2}}}
$$

Where $\rho_{i}$ is the rank correlation coefficient between the expression of miRNA $A_{i}$ and gene in the $\mathrm{i}_{\text {th }} \mathrm{mGl}$. The ranks of miRNA $\mathrm{A}_{\mathrm{i}}$ and gene $\mathrm{i}_{\mathrm{i}}$ expression in $k_{\text {th }}$ sample are represented as $r m_{i k}$ and $r g_{i k} . N$ is the total number of samples in a cancer type. To control the false discovery rate (FDR), $\mathrm{p}$-values of correlations for mGls in each cancer type were further adjusted by Benjamini \& Hochberg method (14). Finally, mGls with $\rho<0$ and FDR<0.05 were considered as functional and reliable regulations in each cancer type.

\section{Validation of identified mGls}

To validate the mGls identified from cancer samples, we built a benchmark dataset by collecting various sources of experimentally validated mGls. Both validated positive and negative mGls were collected from mirRecords (15), miRTarBase (16), TargetMiner 
(17) and TarBase (18) (see supplementary table). After mapping gene IDs to approved HGNC gene symbols (19) and miRBase IDs (20) and integrating redundant mGls from different sources, we finally retrieved 5577 validated mGls including 5139 positive mGls which are validated as functional and 438 negative mGls which are validated as nonfunctional (see supplementary table). Considering the incomplete for both benchmark and our predicted datasets, 166 common mGls involved in our predictions were extracted for evaluation. Contingence table of prediction result was listed in Figures, meanwhile Fisher's exact tests were calculated based on the number of true positive (TP), true negative (TN), false positive (FP) and false negative (FN). Moreover, evaluations of performance were also calculated as below:

$$
\begin{aligned}
& \text { Precision }=T P /(T P+F P) \\
& \text { Recall }=T P /(T P+F N) \\
& F_{1}=2^{*} \text { Precision }{ }^{*} \text { Recall } /(\text { Precision }+ \text { Recall }) \\
& \text { Accuracy }(A C C)=(T P+T N) /(T P+F N+F P+T N)
\end{aligned}
$$

\section{Cancer mutations}

The cancer mutation dataset of 33 cancer types was achieved from "Multi-Center Mutation Calling in Multiple Cancers" (MC3) and was produced using six different algorithms on data from TCGA. 3518261 mutations in 9819 cancer samples were included in our analysis.

\section{Mutation probability}

We mapped cancer mutations to the specified region by bedtools (21). To calculate the mutation probability in a genomic region, we firstly defined mutation rate of mut $_{i}$ as: $R\left(m u t_{i}\right)=N\left(m_{i} t_{i}\right) / N$, where $N\left(\right.$ mut $\left._{i}\right)$ is the number of samples with mutation in a cancer and $N$ is the number of all samples in a cancer. Then mutation probability of a region $r$ was calculated as:

$$
\mathrm{P}(\mathrm{r})=\sum_{r}^{m u t i} R(\text { mut } i) / \text { length }(r) \text {. }
$$

In brief, $P(r)$ was calculated by summing up the mutation rate of all mutations in the region $r$ and normalized by the length of region. In this way, we could compare the level of mutation probability for regions with different lengths and different number of 
mutations. To evaluate the level of mutation probability in mature miRNAs, the mature miRNA regions, seed regions (2-8 bp of each miRNA), upstream and downstream flanking 50bp regions of mature miRNAs were calculated for comparison.

\section{Significance of mutation exclusivity on mGI}

For each mutated mGl, mutations could act on either the miRNA or target gene, or both of them. In this case, we classified mutated mGls as miRNA-mutation mGls, targetmutation mGls and dual-mutation mGls. To find out whether the mutations on mGls work in a synergetic way or in an exclusive way, we evaluated the mutation exclusivity of mGls based on the occurrence of dual-mutation mGls. In this way, mutation exclusivity on miRNA-gene pair was tested by hypergeometric distribution based on the number of all mutated mGls, miRNA-mutation mGls, target-mutation mGls and dualmutation mGls in each cancer type.

$$
p(X \leq d)=F(X \mid N, K, M)=\sum_{X=0}^{d} \frac{\left(\begin{array}{l}
K \\
X
\end{array}\right)\left(\begin{array}{l}
N-K \\
M-X
\end{array}\right)}{\left(\begin{array}{l}
N \\
M
\end{array}\right)}
$$

Where $\mathrm{N}$ is the number of mutated mGls, $\mathrm{K}$ is the number of mGls with miRNA mutation which equals $m+d, M$ is the number of mGls with target mutation which equals $t+d$ and $X$ is the number of dual mutation mGls. In the case, $m$ is the number of miRNA exclusively mutated mGls, $t$ is the number of target exclusively mutated mGls and $d$ is the number of dual-mutation mGls.

\section{Identification of driver mutations on $\mathrm{mGl}$}

For each $\mathrm{mGl}_{\mathrm{j}}$ identified in a type of cancer $_{\mathrm{i}}$, firstly all the mutations within mature miRNA $_{j}$ and binding sites of target gene ${ }_{j}$ were considered as candidate driver mutations. For each mutation $_{\mathrm{k}}$ on $\mathrm{mGl}_{\mathrm{j}}$, we searched samples with mutation $_{\mathrm{k}}$ in cancer $_{\mathrm{i}}$ and integrated them with gene expression of miRNA $A_{j}$ and gene $e_{j}$ in mutated samples. To identify the driver mutations that could affect miRNA-mRNA binding, we assumed the driver mutation on either miRNA or target site could alter the inhibitory role of miRNA

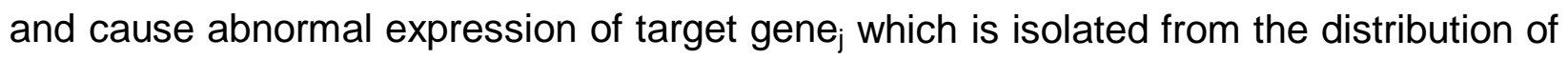
non-mutated control samples. Here two sets of control samples were considered, thus non-mutated cancer samples in 25 cancer types and non-mutated normal samples in 5 
cancer types. For each mutated $\mathrm{mGl}_{\mathrm{j}}$ in cancer $_{\mathrm{i}}$, we used the linear regression model $\mathrm{L}_{\mathrm{j}}$ to fit the expression distribution of control samples and calculated the prediction interval with a probability of 0.95 .

$$
L_{j:} \quad g_{j} \sim \beta_{0}+\beta_{1}{ }^{*} m_{j}
$$

Where $g_{j}$ and $m_{j}$ are the expression of gene ${ }_{j}$ and miRNA $_{j}$ among control samples. $\beta_{0}$ and $\beta_{1}$ are estimated parameters trained by expression of control samples, where only the models with significant $p$ value $(p<0.05)$ are considered as successful. In this way, the control samples should fall into the predictive confident interval while samples with driver mutations on $\mathrm{mGl}_{\mathrm{j}}$ should fall out of it.

\section{Minimum free energy (MFE) change by driver mutations on $\mathrm{mGl}$}

Minimum free energy (MFE) can be used to evaluate the strength of the hybridization between miRNA and target mRNA. The lower the free energy is, the more stable a miRNA could bind to a target mRNA. In this study, we calculated the minimum free energy (MFE) for wild-type mGls and driver-mutated mGls using RNAhybrid (22). The energy change of $\mathrm{mGl}$ altered by driver mutation is defined as:

$$
\Delta E=M F E_{\text {mut }}-M F E_{\text {wild }}
$$

The lower the $\Delta E$ is, the more stable it is for the binding of miRNA-mRNA. Thus it can be more easily for the miRNA to carry out its inhibitory function and downregulate the expression of target gene more effectively.

When calculating the proportions of $\mathrm{mGl}$ with changed or unchanged MFE, we defined mGls with $|\Delta E|$ in top $30 \%$ as energy changed and all the other $70 \%$ as unchanged. Similarly for the gene expression, mGls with $\mid \Delta$ Expression | in top $30 \%$ was defined as expression changed and the others as unchanged.

\section{mGI network analysis}

Network visualization was conducted by the software Cytoscape (23). The layout of driver network was grouped by the classification of driver gene nodes which are "mutated miRNAs", "non-mutated miRNAs", "mutated genes" and "non-mutated genes". Node degree is the number of interactions for each node in the global network. 


\section{Cancer hallmark and enrichment}

To investigate the functional importance of the driver genes and mutations, functional enrichment analysis of the driver genes was carried out to investigate whether they were enriched in cancer hallmarks. The gene list of each cancer hallmark was obtained from one of the previous studies. We used hypergeometric test for exploring the statistical significance and the p-value was computed as follow:

$$
p(X \geq t)=1-F(X \mid N, K, M)=1-\sum_{X=0}^{t} \frac{\left(\begin{array}{l}
K \\
X
\end{array}\right)\left(\begin{array}{l}
N-K \\
M-X
\end{array}\right)}{\left(\begin{array}{l}
N \\
M
\end{array}\right)}
$$

Where $\mathrm{N}$ is the number of genes in the whole genome, of which $\mathrm{K}$ genes were involved in the functional category under investigation (such as cancer hallmarks), and the number of candidate driver genes is $\mathrm{M}$, of which $\mathrm{t}$ genes were involved in the functional category.

\section{Differential expression analysis}

Differential expression analysis was carried out for all genes in cancer types having both tumor and normal samples. Count of raw reads in each gene was downloaded in 'HTSeq-Counts' format from TCGA. R package 'DESeq2' was used to carry out differential analysis. Genes with reads count smaller than 10 in total samples were filtered out. Finally, genes with $\mid$ fold change $\mid>2$ and adjusted $p$ value $<0.05$ were considered as significantly differentially expressed.

\section{Random permutation test for differentially expressed genes}

For a given set of $\mathrm{N}$ genes, we calculated the proportion of significantly down-regulated, up-regulated and non-differentially expressed genes, which are denoted as $\mathrm{D}, \mathrm{U}$ and $\mathrm{M}$. As the definition, $D+U+M=1$. To test whether the observed values of $D, U$ and $M$ are significant, we randomly picked $\mathrm{N}$ genes from all genes in all cancer types and calculated the proportions of them as $d, u$ and $m$ in each random case. By repeating 10000 times, we got the random distribution of variable $\mathrm{d}, \mathrm{u}$ and $\mathrm{m}$. By comparing the $\mathrm{D}$, $U$ and $M$ with the random distributions of $d, u$ and $m$, we got the significance for each classification of differentially expressed genes. 


\section{The web-based resource}

The results in this work were organized in a website resource, "CanVar-mGl" (cancer variant $\mathrm{mGl}$ ). The website was implemented with Perl, JavaScript and HTML and userfriendly accessible.

\section{Results}

\section{Integrative method for construction of pan-cancer miRNA regulatory networks}

To analyze miRNA-gene regulation across cancer types, we proposed a computational method for identifying active miRNA-gene regulation in specific cancer types (Figure 1A). In brief, high-throughput sequencing of immunoprecipitated RNAs after cross-link (CLIP-Seq) to AGO proteins was used to identify endogenous genome-wide interaction maps for miRNAs (12) and bioinformatics tools were developed to infer specific target sites each miRNA could bind to $(11,24,25)$. In a previous study, Agarwal et al. showed that many non-canonical sites detected by cross-linking methods did not mediate gene repression despite their ability to bind miRNAs while the vast majority of functional sites were canonical and could be identified by TargetScan (v7.0) (11). In this study, we integrated AGO CLIP-Seq data from starBase v2.0 (13) with TargetScan 7.0 (11) and derived 279,924 miRNA-gene interactions (mGls) among 2,586 mature miRNAs and 4,198 target genes as a global reference network. After the inference of the global mGI network, we integrated the miRNA and gene expression profiles in specific cancer types to identify active miRNA-gene regulation. Only negatively correlated miRNA-gene pairs $(\mathrm{FDR}<0.05)$ were kept for further analysis. In total, 107,068 unique mGls were obtained from 30 of 33 cancer types. Next, we validated our results using a benchmark dataset of experimental results collected from various sources. The performance of our predictions was: recall rate $=93.5 \%$, precision $=94.1 \%, \mathrm{~F} 1$ score $=93.8 \%$, accuracy $=88.6 \%$. In addition, our predictions exhibited a significant overlap with the benchmark dataset (Figure 1B, odds ratio=6.36 and $p=0.015$ ). These results indicate that integration of multi-omics datasets enables us to identify cancer type-specific active miRNA-gene regulation at the systems level. 
Comparing miRNA-gene regulatory networks across cancer types, we identified marked rewiring in the miRNA regulatory programs among different cancer types, with a unique 'on/off' switch depending upon the cancer type. We found that $242.47 \%$ miRNA-gene regulation occurred only in one cancer type (Figure 1C) but only $1.55 \%$ miRNA-gene regulation was observed in greater than 10 cancer types. The low conservation of miRNA-gene regulation could be explained in part by the cancer-specific expression of miRNAs or target genes. We also observed that the distribution of cancer frequent (common) mGls ( $\mathrm{N}>=10$ cancer types) was more homogeneous than that of total mGls (Figure S1A and S1B). This indicates although the proportion of frequent mGls is small, they are relatively stable across cancer types. In contrast, the number of cancer specific mGls increased as the total number of mGls grew (Figure S1C). Moreover, we performed functional enrichment analysis using the target genes that were observed in cancer frequent mGls. We found that these genes were involved in general cancerrelated functions, including FoxO signaling pathway, TGF-beta signaling pathway and cell adhesion related functions (Figure 1D). Furthermore, hierarchical clustering analysis was conducted based on the occurrence of cancer frequent mGls. Several cancer types with similar origins were clustered together (Figure S1D). OV (ovary) and CESC (cervix) were clustered into gynecologic system, showing low occurrences of cancer frequent mGIs. KIRP and KIRC (kidney) were clustered into urologic system, showing medium occurrences of frequent mGIs. COAD, READ (colorectal) and LUAD, LUSC (lung) were separately clustered into gastrointestinal and thoracic systems, respectively, showing relatively high occurrences of frequent mGls. All these results indicate the intricate functional roles of miRNA regulation in cancer. Taken together, our integrative analysis reveals cancer-specific miRNA-gene regulation, providing a valuable resource for mechanistic investigation of the function of miRNAs in cancer.

\section{miRNA mutations and target gene mutations in cancer}

miRNAs play important roles in the development and progression of cancer. Several miRNA-associated mutations have been identified before, but systematic analysis of mutational landscape on miRNA regulation is still lacking. In this study, cancer somatic mutations obtained from the TCGA project were mapped to miRNAs and their target 
genes, or other regions in the human genome. We found that the mutation probability in miRNAs was greater than that in mRNAs (Figure 2A, $\mathrm{p}<2.2 \mathrm{e}-16$, Wilcoxon rank-sum test; Figure S2). Moreover, we found that the genomic regions of miRNAs also had a higher mutation probability than upstream or downstream flanking regions (Figure 2A, $\mathrm{p}<2.2 \mathrm{e}-16)$. Specifically, the mutation probability of the seed regions within miRNAs was greater than other parts within miRNAs or the flanking regions $(p<2.2 e-16)$, suggesting the seed regions of miRNAs are required for miRNA regulation. In addition, we identified thousands of mutations located in target coding genes of miRNAs. Compared with the flanking regions, the miRNA binding sites in target genes exhibited a higher mutation probability (Figure $2 \mathrm{~B}$ ). These results indicate that mutations are likely to occur in the genomic regions that are critical for miRNA binding.

To further explore if these mutations could play a functional role in perturbing miRNAgene regulation, we estimated the functional impact of all somatic mutations identified in cancer. Several methods have been proposed to assess the effects of mutations on protein function, and these methods are complementary to each other. We herein used Combined Annotation-Dependent Depletion (CADD) (Kircher et al., 2014), which is a framework that integrates multiple annotations into one metric, to explore the functional impact of mutations in target genes of miRNAs. For each cancer type, we randomly selected the same number of mutations as background controls. We then compared the CADD scores of mutations in target genes with those of randomly selected mutations. In the majority (23/31) of cancer types, mutations in target genes had significantly higher scores (Wilcoxon rank-sum test $\mathrm{p}<0.05$ ) than random controls (Figure 2C), suggesting that the identified miRNA target mutations could have deleterious effects in cancer. Evolutionary conservation of mutated residues has also been demonstrated to reflect the functional importance of the mutational event (Watson et al., 2013). We therefore explored the conservation feature of the mutations in target genes. We found that positions harboring target gene mutations were more likely to be conserved than positions harboring randomly selected mutations in most (29/31) cancer types (Figure 2D). All these results indicate that there are widespread mutations enriched within 
miRNA sites and their target genes, which could functionally perturb the binding of miRNAs, thus playing critical roles in cancer.

\section{Mutually exclusive mutations in miRNA-gene regulation}

It has been observed that sets of genes that are co-involved in the same cancer pathways tend not to harbor mutations together in the same patient, which is called mutual exclusivity $(26,27)$. However, it remains elusive if the mutual exclusivity hypothesis could be extended in the context of miRNA regulation. We therefore assessed whether the same cancer patients could simultaneously carry miRNA mutations and target gene mutations. To do so, we calculated the number of miRNAgene interactions (mGls) for three mutation perturbation models. The first one is that mutations located only in miRNAs to perturb the interactions; the second one is that mutations located only in target genes to perturb the interactions; and the third one is that mutations are located in both miRNAs and their target genes (Figure 3A). We found that a vast majority of mGls harbored miRNA mutations or target mutations, and only a small fraction of mGls had both miRNA and target mutations (Figure 3B). In addition, we found that patients in different cancer types exhibited different regulatory patterns (Figure 3C; Figure S3). For example, the majority of patients with ACC, LAML, PAAD and READ carried miRNA mutations. In contrast, TGCT, THYM, PCPG patients were likely to have target binding site mutations. In summary, we found that the mutual exclusivity of miRNA versus target mutations was more significant across cancer types than expected by chance (Figure $3 C, p<0.05$ for $23 / 25$ cancer types).

Specifically, we identified mutually exclusive mGls that had been reported in various types of cancer. For example, the let-7 family is a conserved family of miRNAs and the deregulation of this family was observed in many cancer types, such as breast cancer, lung cancer and melanoma. Here, several mutations were observed in let-7f-1-3p, let$7 a-5 p$ and let-7b-3p. In addition, several mutations located within target genes were also observed in patients, such as LIFR, SPIRE1 and E2F3. However, no patient was found with both miRNA mutations and target gene mutations. These observations suggest that the mutations selectively target miRNAs or target genes to perturb miRNA- 
gene regulation in cancer, which is consistent with the pathway redundancy model in cancer. Next, we explored the signaling pathways that were likely perturbed by these mutations. As the hallmarks of cancer comprise biological capabilities acquired during the multistep development of human tumors, we evaluated whether these mutations could possibly perturb cancer hallmark related functions. As shown in Figure 3D, we found that the mutated miRNA target genes were significantly enriched in various types of cancer hallmarks, such as regulation of cell proliferation and DNA repair. Together, these results indicate that these mutually exclusive mutations within miRNAs or target genes likely perturb cancer hallmark related functions.

\section{Identification of driver mutations on mGls}

Distinguishing driver mutations from passenger mutations in individual genomes is a big challenge in cancer genomic studies. In this study, we integrated the mGl regulatory networks with target gene transcriptomic networks to identify candidate driver mutations in each cancer type. Our hypothesis was that candidate driver mutations were likely to perturb mGls which could be reflected by a significant change in the expression levels of target genes (Figure $4 \mathrm{~A}$ ). We thus used a linear regression model to fit the expression distribution among non-mutated control samples and identified driver mutations that elicited significant deviations from the normal correlation between miRNAs and their target genes (see details in methods). In total, we identified 89 driver mutations across 14 cancer types (Figure 4B; Figure S4). Among 89 driver mutations, 75 of them occurred in target genes and 14 of them occurred in miRNAs. In addition, 9 of 75 driver mutations in target genes had indeed been characterized as cancer mutations in the Catalogue of Somatic Mutations in Cancer (COSMIC) database and in this study we inferred their possible functions through miRNA regulatory networks.

Mutations at $\mathrm{mGl}$ interfaces could impair the binding of miRNAs to target genes by changing the minimum free energy (MFE) of binding sites, thus further tuning the regulation of target gene expression. In this study, we calculated the changes of MFE using RNAhybrid (22) and changes of target gene expression between wild-type and mutated mGls. By comparative analysis of different types of mGls, we found that the 
energy changes were significantly correlated with the changes in target gene expression levels by Fisher's exact test (Figure 4C, $p=0.002$, odds ratio=3.3). We next speculated that if driver mutations disrupt miRNA-target binding, one would expect the repression of target gene expression by miRNAs to be reversed (i.e., increased). Consistently, we found that target genes with increased expression (e.g., top 5\%) showed a significantly higher alteration of MFE than those with decreased expression (e.g., bottom 5\%) by Wilcoxon rank-sum test ( $p=0.038$, Figure 4D). Together, these results suggest that driver mutations are likely to perturb mGls in cancer through their functional influence on miRNA binding affinity to target genes.

\section{mGI network perturbations by driver mutations}

To analyze the structure and properties of perturbed mGl networks by driver mutations, we extracted all mGls involving both miRNAs and target genes that harbored driver mutations. As shown in Figure 5A, the bipartite mGl networks had four types of node components: mutated miRNAs, non-mutated target genes, non-mutated miRNAs and mutated target genes. mGls could be perturbed by mutations in the miRNAs (mostly interactions between mutated miRNAs and non-mutated targets), or perturbed by mutations in the target genes (mostly interactions between non-mutated miRNAs and mutated targets). We then examined the functional pathways impacted by the driver mutation-induced $\mathrm{mGl}$ network perturbations. Through analysis of cancer hallmark signatures, we found that perturbed target genes were enriched in "insensitivity to antigrowth signals" and "self-sufficiency in growth signals" (Figure 5B). These two functions could help explain the increased growth and escaping from antigrowth signals in the cancer cells carrying these driver mutations. Furthermore, we investigated the expression profiles of all the target genes in the perturbed $\mathrm{mGl}$ networks. As shown in Figure 5C and 5D, 83.3\% (40/48) of the mutated target genes in perturbed networks were differentially expressed in at least one cancer type, and 68.9\% (31/45) of the nonmutated target genes (but targeted by mutated miRNAs) were differentially expressed in at least one cancer type. These results indicate that the target genes perturbed by driver mutations are associated with cancer development. 
Intriguingly, for the miRNAs carrying driver mutations (shown in top left of Figure 5A), all of them were from the let-7 family including hsa-let-7a-2-3p, hsa-let-7a-3p, hsa-let-7a$5 p$, hsa-let-7b-3p, hsa-let-7c-3p, hsa-let-7c-5p, hsa-let-7f-1-3p, hsa-let-7f-2-3p, hsa-let$7 f-5 p$ and hsa-let-7g-3p. As a well-known miRNA family with functions of tumor suppression, aberrant regulation of the let-7 family had been reported in many cancer types (28). These included increased cellular proliferation in lung cancer (29), increased proliferation and migration in liver cancer (30), increased invasion and metastasis in gastric adenocarcinoma, lymph node metastasis in breast cancer (31) and so on. Our study further recaptures the functional importance of the let-7 family in cancer by pointing out that the let-7 family could rewire molecular signaling through mutations within miRNAs themselves to dysregulate their targets.

\section{Driver mutations primarily target tumor suppressor genes}

To take a close investigation on the mutated target genes, we made a volcano plot for them in their matched cancer types and found $26.3 \%$ of the mutated targets were downregulated (Figure 6A; upper left region). To explore the significance of this proportion, we randomly picked the same number of genes as the mutated target gene set in all cancer types 10,000 times and generated the random distribution as a background control. We found that the observed proportion of down-regulated genes in reality was significantly higher than expected by chance (Figure 6B). In addition, the proportions of up-regulated and non-differentially expressed genes were not significant (Figure S5) which supports the conclusion that mutated target genes are significantly downregulated.

By curation from literature, we found that most of the down-regulated mutated target genes were tumor suppressor genes (Figure 6C). The top mutated target genes were all cancer related and mostly annotated as inhibiting cancer progression. We further extracted the perturbed networks of these tumor suppressors (Figure 6D). Upstream miRNAs (red nodes) and their interactions with these tumor suppressors were perturbed by driver mutations. While the other target genes of these upstream miRNAs were unperturbed, no significant functional pathways were enriched for unperturbed targets. 
In the perturbed tumor suppressor networks, all the target genes involved and most of the miRNAs had previous evidence to be associated with cancer. For example, LIFR could confer a dormancy phenotype in breast cancer cells and also negatively regulate the metastasis of pancreatic cancer cells $(32,33)$. RBM24 was demonstrated to suppress cancer progression in nasopharyngeal carcinoma and also reported as a novel player in the p53 pathway $(34,35)$. KLF15 could inhibit the cell proliferation in gastric cancer (36). AKAP12 was reported as a tumor suppressor in glioblastoma and prostate cancer, and regulated by miRNA pathways (37-39). CBX7 was reported as a tumor suppressor in colon, thyroid carcinomas, glioblastoma and pancreatic cancer (3941). It could play its role by modulating the KRAS gene and could be regulated by miRNAs $(39,40)$. For the upstream miRNAs of these tumor suppressors, hsa-miR6881-3p was known to play its role through the p53-mediated ceRNAs network in hepatocellular carcinoma (42). hsa-miR-15b-5p was used to distinguish human ovarian cancer tissues from normal tissues (43). hsa-miR-193b-3p was found differentially expressed in hepatocellular carcinoma (44). has-miR-15a-5p was shown to decrease melanoma cell viability and could cause cell cycle arrest at the G0/G1 phase (45). hsamiR-769-3p was differentially expressed in non-small cell lung cancer (NSCLC) patients harboring EGFR mutations (46). In conclusion, the mutated driver target genes perturbed through miRNA pathways play their roles probably as tumor suppressors and are down-regulated in many cancer types thus accelerating the growth of cancer cells.

\section{CanVar-mGl: a web-based resource for mutation-mediated mGl network perturbations in cancer}

To help researchers apply the principles described in this work to any mGls or mutations of interest, we developed a comprehensive and interactive web resource, CanVar-mGI. The features provided in the resource, which will be continuously updated, should serve as a guide for biologists interested in identifying miRNA-gene regulation in specific cancer types and understanding the consequences of mutations that perturb specific miRNA-gene interactions in cancer. All the dataset could also be downloaded 
for further analysis of the functional effect of mutations and miRNA-gene regulation (Figure S6).

\section{Discussion}

In this study, we derived de novo cancer specific miRNA-gene interactions with high quality by integration of in silico predictive models, AGO CLIP-Seq data, and transcriptome networks. We built out these $\mathrm{mGl}$ networks of various cancer types as a framework for pan-cancer analysis of the functional consequences of somatic mutations on mGls. Further analysis showed that the mutations on mGls exhibited a mutually exclusive pattern. Mutual exclusivity was previously observed for mutations in gene members of the same pathways. Yeang et al. found that when a member of a pathway was altered, the selection pressure on the other members was diminished or even nullified (47). As a result, significantly less overlap in mutations of the other genes was expected, creating a mutually exclusive pattern between their alterations. Supporting this expectation, it was previously shown that some functionally related genes were mutated in a mutually exclusive manner in thyroid tumors $(48,49)$ and in leukemia $(50)$. In our study, we observed this pattern on miRNA-gene interactions in most of the cancer types.

To identify driver mutations that can perturb the mGls, we compared the samples with mutations on mGls with the fitted expression distribution in non-mutated samples and evaluated the deviations of each candidate mutations. After identification of driver mutations, we analyzed the alteration of minimum free energy and target gene expression between driver mutant and wild type mGls. We found that the target gene expression was associated with the alteration of minimum free energy, indicating that the driver mutations could perturb mGls by influencing mGl binding sites.

The genetic changes that contribute to cancer tend to affect three main types of genes-proto-oncogenes, tumor suppressor genes, and DNA repair genes which are called "drivers" of cancer. In our analysis, we have shown that miRNA related driver mutations tend to play their roles on tumor suppressor genes by modulating the miRNA- 
gene interactions. Tumor suppressor genes are involved in controlling cell growth and division. Cells with certain alterations in tumor suppressor genes may divide in an uncontrolled manner. Concordantly, our analyses show that the identified target genes in the perturbed miRNA regulatory networks are enriched in the cancer hallmarks of "insensitivity to antigrowth signals" and "self-sufficiency in growth signals" which are consistent with the function of tumor suppressors. In this study, while several known tumor suppressors have been revealed and perturbed by miRNA related driver mutations, such as LIFR, RBM24, PTGER3, several identified novel mutated driver genes such as LDLRAD4, AKAP5, KLHL6 and so on, could become potential biomarkers and therapeutic targets for tumor suppression.

\section{Disclosure of Potential Conflicts of Interest}

No potential conflicts of interest were disclosed by the authors.

\section{Acknowledgements}

We were also grateful to contributions from TCGA Research Network Analysis Working Group. We also acknowledge the Biomedical Research Computing Facility at UT Austin and Texas Advanced Computing Center (TACC) for high-performance computing assistance.

\section{Grant Support}

This work was supported by the National Institutes of Health grant [K22CA214765 to S.Y.] and Komen Foundation grant [CCR19609287 to S.Y.]. 


\section{References}

1. E. D. Pleasance et al., A comprehensive catalogue of somatic mutations from a human cancer genome. Nature 463, 191-196 (2010).

2. N. McGranahan et al., Clonal status of actionable driver events and the timing of mutational processes in cancer evolution. Sci Transl Med 7, 283 ra254 (2015).

3. A. Esquela-Kerscher, F. J. Slack, Oncomirs - microRNAs with a role in cancer. Nat Rev Cancer 6, 259-269 (2006).

4. E. R. Gamazon et al., Genetic architecture of microRNA expression: implications for the transcriptome and complex traits. Am J Hum Genet 90, 1046-1063 (2012).

5. A. Bhattacharya, J. D. Ziebarth, Y. Cui, SomamiR: a database for somatic mutations impacting microRNA function in cancer. Nucleic Acids Res 41, D977-982 (2013).

6. A. Bhattacharya, Y. Cui, SomamiR 2.0: a database of cancer somatic mutations altering microRNA-ceRNA interactions. Nucleic Acids Res 44, D1005-1010 (2016).

7. L. Bao et al., PolymiRTS Database: linking polymorphisms in microRNA target sites with complex traits. Nucleic Acids Res 35, D51-54 (2007).

8. J. D. Ziebarth, A. Bhattacharya, A. Chen, Y. Cui, PolymiRTS Database 2.0: linking polymorphisms in microRNA target sites with human diseases and complex traits. Nucleic Acids Res 40, D216-221 (2012).

9. A. Bhattacharya, J. D. Ziebarth, Y. Cui, PolymiRTS Database 3.0: linking polymorphisms in microRNAs and their target sites with human diseases and biological pathways. Nucleic Acids Res 42, D86-91 (2014).

10. S. Stegeman et al., A Large-Scale Analysis of Genetic Variants within Putative miRNA Binding Sites in Prostate Cancer. Cancer Discov 5, 368-379 (2015).

11. V. Agarwal, G. W. Bell, J. W. Nam, D. P. Bartel, Predicting effective microRNA target sites in mammalian mRNAs. Elife 4, (2015).

12. J. Konig, K. Zarnack, N. M. Luscombe, J. Ule, Protein-RNA interactions: new genomic technologies and perspectives. Nat Rev Genet 13, 77-83 (2012).

13. J. H. Li, S. Liu, H. Zhou, L. H. Qu, J. H. Yang, starBase v2.0: decoding miRNA-ceRNA, miRNA-ncRNA and protein-RNA interaction networks from large-scale CLIP-Seq data. Nucleic Acids Res 42, D92-97 (2014).

14. Y. Benjamini, Y. Hochberg, Controlling the False Discovery Rate - a Practical and Powerful Approach to Multiple Testing. Journal of the Royal Statistical Society Series BMethodological 57, 289-300 (1995).

15. F. F. Xiao et al., miRecords: an integrated resource for microRNA-target interactions. Nucleic Acids Research 37, D105-D110 (2009). 
16. C. H. Chou et al., miRTarBase 2016: updates to the experimentally validated miRNAtarget interactions database. Nucleic Acids Research 44, D239-D247 (2016).

17. S. Bandyopadhyay, R. Mitra, TargetMiner: microRNA target prediction with systematic identification of tissue-specific negative examples. Bioinformatics 25, 2625-2631 (2009).

18. G. L. Papadopoulos, M. Reczko, V. A. Simossis, P. Sethupathy, A. G. Hatzigeorgiou, The database of experimentally supported targets: a functional update of TarBase. Nucleic Acids Research 37, D155-D158 (2009).

19. K. A. Gray, B. Yates, R. L. Seal, M. W. Wright, E. A. Bruford, Genenames.org: the HGNC resources in 2015. Nucleic Acids Research 43, D1079-D1085 (2015).

20. A. Kozomara, S. Griffiths-Jones, miRBase: annotating high confidence microRNAs using deep sequencing data. Nucleic Acids Res 42, D68-73 (2014).

21. A. R. Quinlan, I. M. Hall, BEDTools: a flexible suite of utilities for comparing genomic features. Bioinformatics 26, 841-842 (2010).

22. J. Kruger, M. Rehmsmeier, RNAhybrid: microRNA target prediction easy, fast and flexible. Nucleic Acids Research 34, W451-W454 (2006).

23. P. Shannon et al., Cytoscape: a software environment for integrated models of biomolecular interaction networks. Genome Res 13, 2498-2504 (2003).

24. M. D. Paraskevopoulou et al., DIANA-microT web server v5.0: service integration into miRNA functional analysis workflows. Nucleic Acids Res 41, W169-173 (2013).

25. D. Betel, A. Koppal, P. Agius, C. Sander, C. Leslie, Comprehensive modeling of microRNA targets predicts functional non-conserved and non-canonical sites. Genome Biol 11, R90 (2010).

26. O. Babur et al., Systematic identification of cancer driving signaling pathways based on mutual exclusivity of genomic alterations. Genome Biol 16, 45 (2015).

27. R. K. Thomas et al., High-throughput oncogene mutation profiling in human cancer. Nat Genet 39, 347-351 (2007).

28. T. Wang et al., Aberrant regulation of the LIN28A/LIN28B and let-7 loop in human malignant tumors and its effects on the hallmarks of cancer. Mol Cancer 14, 125 (2015).

29. J. Takamizawa et al., Reduced expression of the let-7 microRNAs in human lung cancers in association with shortened postoperative survival. Cancer Res 64, 3753-3756 (2004).

30. J. Ji et al., Let-7g targets collagen type I alpha2 and inhibits cell migration in hepatocellular carcinoma. J Hepatol 52, 690-697 (2010).

31. X. Hu et al., The heterochronic microRNA let-7 inhibits cell motility by regulating the genes in the actin cytoskeleton pathway in breast cancer. Mol Cancer Res 11, 240-250 (2013). 
32. R. W. Johnson et al., Induction of LIFR confers a dormancy phenotype in breast cancer cells disseminated to the bone marrow. Nat Cell Biol 18, 1078-1089 (2016).

33. D. Ma et al., Leukemia inhibitory factor receptor negatively regulates the metastasis of pancreatic cancer cells in vitro and in vivo. Oncol Rep 36, 827-836 (2016).

34. W. F. Hua et al., RBM24 suppresses cancer progression by upregulating miR-25 to target MALAT1 in nasopharyngeal carcinoma. Cell Death Dis 7, e2352 (2016).

35. Y. Jiang et al., Rbm24, an RNA-binding protein and a target of p53, regulates p21 expression via mRNA stability. J Biol Chem 289, 3164-3175 (2014).

36. C. Sun et al., KLF15 Inhibits Cell Proliferation in Gastric Cancer Cells via Up-Regulating CDKN1A/p21 and CDKN1C/p57 Expression. Dig Dis Sci 62, 1518-1526 (2017).

37. J. Kang et al., TFAP2C promotes lung tumorigenesis and aggressiveness through miR183- and miR-33a-mediated cell cycle regulation. Oncogene 36, 1585-1596 (2017).

38. P. Peixoto et al., HDAC7 inhibition resets STAT3 tumorigenic activity in human glioblastoma independently of EGFR and PTEN: new opportunities for selected targeted therapies. Oncogene 35, 4481-4494 (2016).

39. H. K. Ko et al., A transgenic mouse model for early prostate metastasis to lymph nodes. Cancer Res 74, 945-953 (2014).

40. W. Wu et al., The malignancy of miR-18a in human glioblastoma via directly targeting CBX7. Am J Cancer Res 7, 64-76 (2017).

41. S. Ni et al., CBX7 suppresses cell proliferation, migration, and invasion through the inhibition of PTEN/Akt signaling in pancreatic cancer. Oncotarget 8, 8010-8021 (2017).

42. Y. Zhang et al., Identification and Analysis of P53-Mediated Competing Endogenous RNA Network in Human Hepatocellular Carcinoma. Int J Biol Sci 13, 1213-1221 (2017).

43. L. Wang et al., A ten-microRNA signature identified from a genome-wide microRNA expression profiling in human epithelial ovarian cancer. PLoS One 9, e96472 (2014).

44. Y. Jiang, H. Gao, M. Liu, Q. Mao, Sorting and biological characteristics analysis for side population cells in human primary hepatocellular carcinoma. Am J Cancer Res 6, 18901905 (2016).

45. C. Alderman, Y. Yang, The anti-melanoma activity and oncogenic targets of hsa-miR15a-5p. RNA Dis 3, (2016).

46. Y. Ma et al., Plasma microRNA alterations between EGFR-activating mutational NSCLC patients with and without primary resistance to TKI. Oncotarget 8, 88529-88536 (2017).

47. C. H. Yeang, F. McCormick, A. Levine, Combinatorial patterns of somatic gene mutations in cancer. FASEB J 22, 2605-2622 (2008).

48. M. Xing et al., Early occurrence of RASSF1A hypermethylation and its mutual exclusion with BRAF mutation in thyroid tumorigenesis. Cancer Res 64, 1664-1668 (2004). 
49. Y. Wang et al., High prevalence and mutual exclusivity of genetic alterations in the phosphatidylinositol-3-kinase/akt pathway in thyroid tumors. J Clin Endocrinol Metab 92, 2387-2390 (2007).

50. N. Carbuccia et al., Mutual exclusion of ASXL1 and NPM1 mutations in a series of acute myeloid leukemias. Leukemia 24, 469-473 (2010).

51. M. I. Love, W. Huber, S. Anders, Moderated estimation of fold change and dispersion for RNA-seq data with DESeq2. Genome Biol 15, 550 (2014). 


\section{Figure legends}

Figure 1. The landscape of miRNA-gene regulatory networks across cancer types.

(A) The workflow to identify genome-wide miRNA-gene regulation in cancer. Firstly, global miRNA-gene interactions were identified by integrating sequence alignment and AGO-GLIP-Seq datasets. Then miRNA and mRNA expression profiles were integrated to identify cancer-specific interactions. Somatic mutations were mapped to mGls to identify candidate driver mutations.

(B) Validation of the predicted miRNA-gene interactions. The numbers in the table show the overlap of predicted mGls and literature-supported mGls. Fisher's exact test was used to evaluate the significance.

(C) The proportion of mGls in different numbers of cancer types.

(D) The KEGG pathway enrichment of miRNA target genes found in more than ten types of cancer.

\section{Figure 2. miRNA and target gene mutations across cancer types.}

(A) The distribution of mutation probability for miRNA-related regions across cancer types, including up-stream, downstream of miRNA regions, miRNAs, seed regions and mRNA regions.

(B) The distribution of mutation probability for target gene-related regions, including upstream, down-stream, miRNA binding sites in targets, and all target mRNA regions.

(C) The average CADD scores for mutations in miRNA target genes and randomly selected genes. Red lines for target genes and blue lines for randomly selected genes. Cancer types marked with red color indicate that the CADD scores for mutations in targets are significantly higher $(p<0.05)$ than other mutations.

(D) The conservation scores for mutations in miRNA target genes and randomly selected genes. Red lines for target genes and blue lines for randomly selected genes. Cancer types marked with red color indicate that the conservation scores for mutations in targets are significantly higher $(p<0.05)$ than other mutations.

\section{Figure 3. The mutual exclusivity of mutations in mGls.}

(A) Three models to illustrate the mutual exclusivity of mutations in mGls. 
(B) The proportion of mGls with different types of mutations. Purple, mGls with miRNA mutations; green, mGls with target mutations; and blue for mGls with both miRNA and target mutations.

(C) Pie plots show the proportion of samples with different types of mutated mGls. I, the proportion of samples with miRNA mutations; II, the proportion of samples with target mutations; III, the proportion of samples with both miRNA and target mutations. Dot plots at the bottom indicate the significance level for mutual exclusivity test.

(D) The cancer hallmark functions enriched by the mutated genes in mGls. The size of the dots represents the significance and the color of the dots represents different types of cancer hallmarks.

\section{Figure 4. The candidate driver mutated mGls across cancer types.}

(A) The linear regression model is used to identify the candidate driver mutated mGls in cancer.

(B) The statistics of driver mutations and driver mGls across cancer types.

(C) The proportion of mGls with energy changed or not for expression changed/unchanged mGls. Orange, energy changed; green, energy unchanged.

(D) The correlation between energy change and expression change.

Figure 5. Network perturbation by driver mutations.

(A) Gene regulatory networks perturbed by driver mutations in mGls. Yellow edges are perturbed mGls and grey edges are unperturbed mGls. Red nodes are mutated miRNAs and orange nodes are non-mutated miRNAs; blue nodes are mutated target genes and cyan nodes are non-mutated genes.

(B) Cancer hallmark enrichment of driver target genes. Hallmarks with odds ratio ( $\mathrm{x}$ axis) $>1$ and $p$-value (y-axis) $<0.05$ are significantly enriched.

(C) Differential expression of mutated driver target genes. Each row represents a driver gene which is differentially expressed in at least one cancer type. Each column represents a cancer type. The occurrence of each point indicates the gene is significantly differentially expressed in that cancer which means the fold change of expression is larger than 2 or smaller than $1 / 2$ and the p value calculated by DESeq2 
(51) is smaller than 0.05 . The red color indicates the gene is up-regulated in cancer samples versus normal samples, while the blue color indicates down-regulation.

(D) Differential expression of non-mutated driver target genes. Legends are the same as above (C).

Figure 6. Tumor suppressors are often targeted in perturbed miRNA regulatory networks.

(A) Volcano plot of mutated target genes in the cancer type where the driver mutation occurs. X-axis shows the log2 value of expression fold change and $Y$-axis shows the value of $-\log 10$ (False Discovery Rate). Each point indicates the gene in the cancer type where the driver mutation is found. The color of each point indicates the cancer type.

(B) Mutated driver genes are significantly down-regulated. Bar plot shows the random distribution of the proportion of down-regulated genes in mutated driver genes. The smoothed curve is the probability density of random distribution. The dashed vertical line indicates the observed proportion of down-regulated genes.

(C) A table showing evidence that many mutated driver genes are tumor suppressors.

(D) Perturbation of tumor suppressor regulatory networks. Yellow edges are perturbed mGls and grey edges are unperturbed mGls. Red nodes are miRNAs and stared blue nodes are mutated target genes. Small genes in left grey box are the targets of these miRNAs which are unperturbed. 


\section{Figure 1}

A

I) Identification of global miRNAgene interactions (mGls) by integrating sequence scanning and AGO-CLIP-Seq datasets

\section{II) Cancer-type specific} miRNA-gene interactions (mGls)

III) Mutation-perturbed mGls and identification of candidate driver mutations

B

Validation of the miRNA-gene interactions

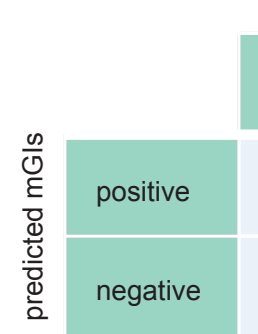

gold standard mGls

\begin{tabular}{|c|c|}
\hline positive & negative \\
\hline 143 & 9 \\
\hline 10 & 4 \\
\hline
\end{tabular}

$\mathrm{OR}=6.36$

p-value $=0.015$

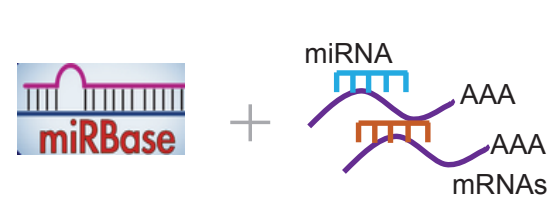

miRNA sequence

(2588 mature miRNAs)

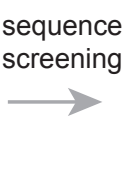

whole human genome

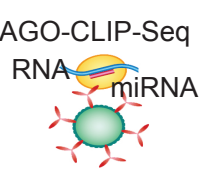

36 datasets

Global miRNA-gene regulations

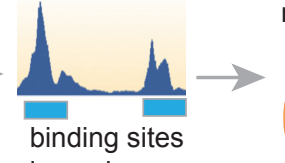

in peaks

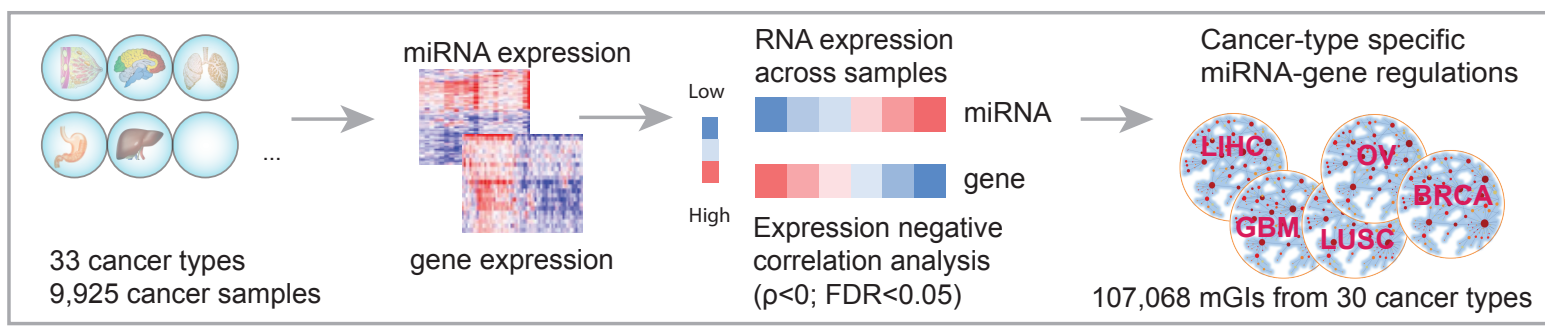

$\begin{aligned} & \text { mapping to miRNA- } \\ & \text { gene regulation } \\ & \text { 10,224 samples }\end{aligned}$
mutation

C

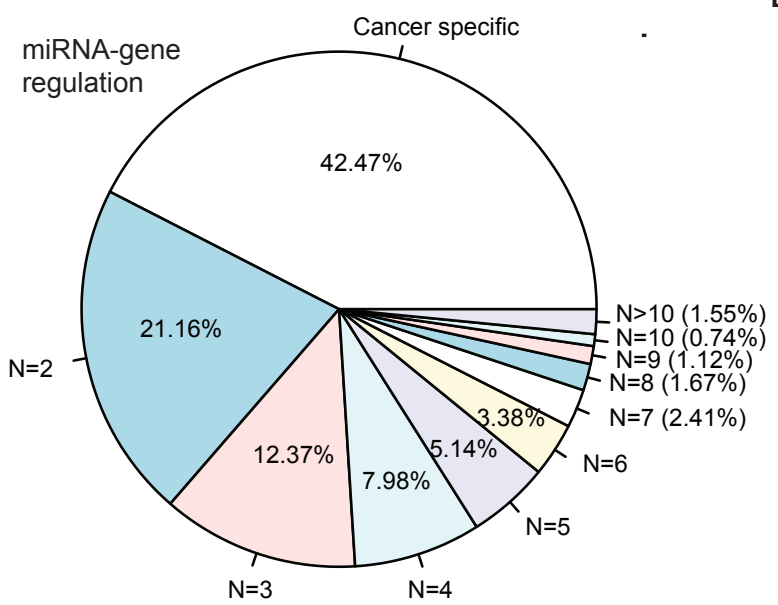

$-\log 2(p-v a l u e s)$

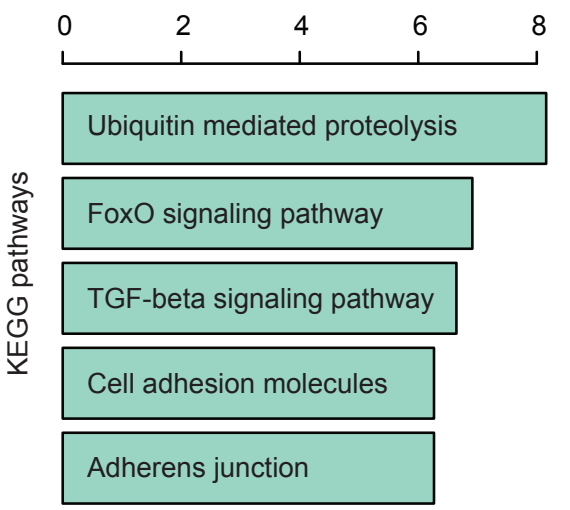


A
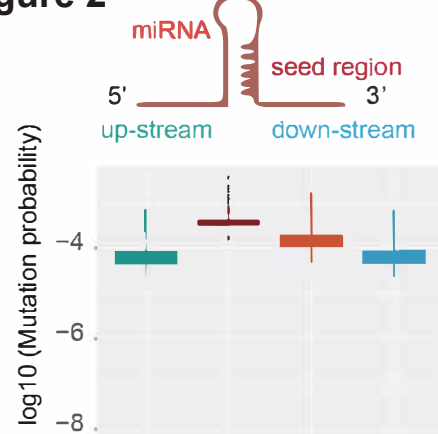

up-Stream miRNA seed region down-stream seed region
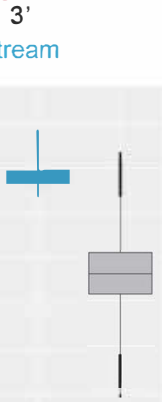

mRNA

B

miRNA

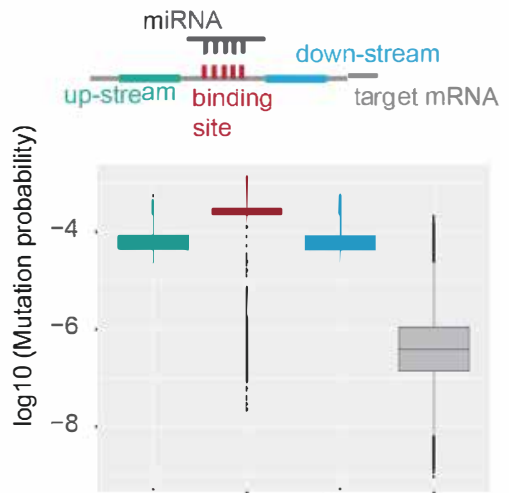

up-stream down-stream

binding site target mRNA

C

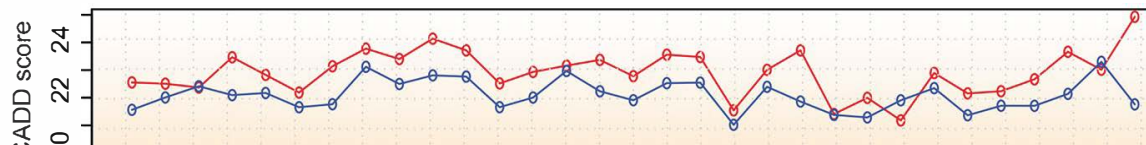

ษั

D

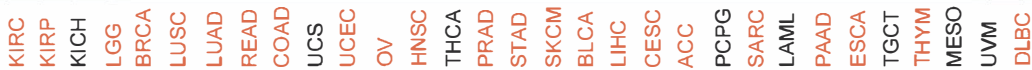

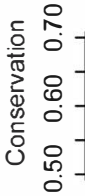

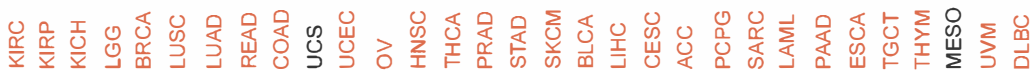

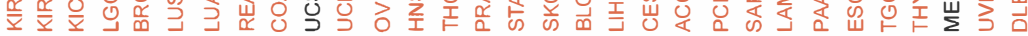


Figure 3

A

\begin{tabular}{|c|c|c|c|}
\hline \multirow{2}{*}{ miRNA } & Model-I & Model-II & Model-III \\
\hline & T市I & TIII & TाTा \\
\hline & ШШ & It L & 111 \\
\hline
\end{tabular}

C

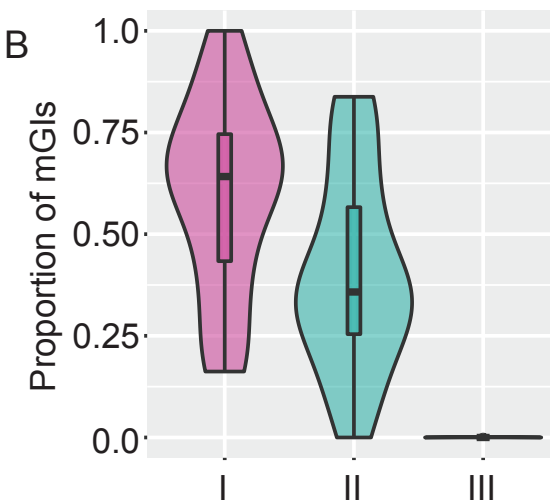

Cancer types

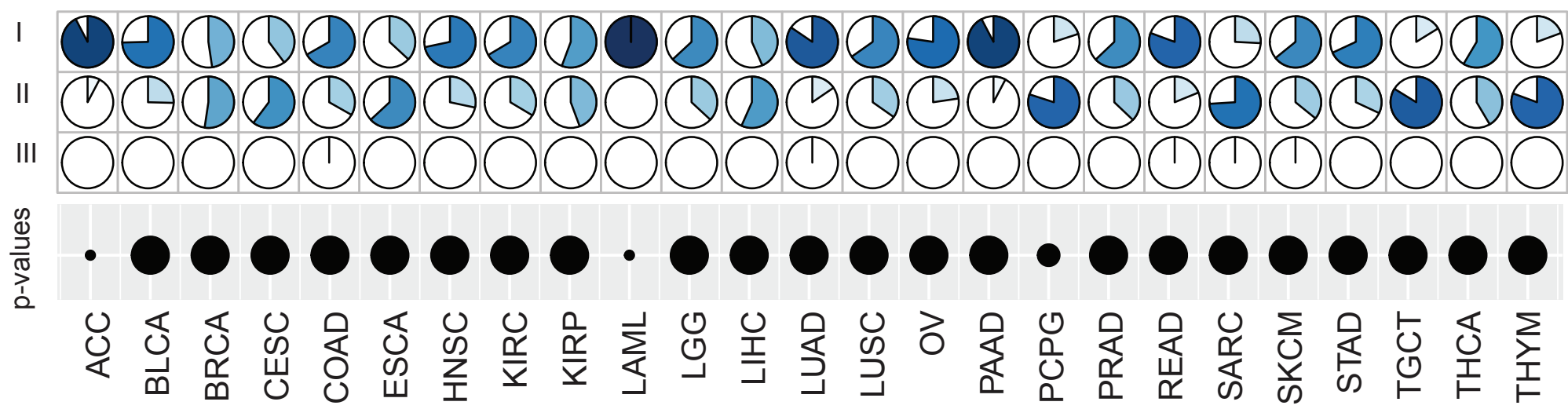

I: mGIs with miRNA mutations II: mGls with target mutations III: mGIs with both mutations

Proportion of mGls

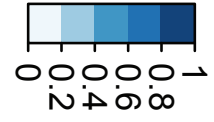

$0.01<p<=0.05 \quad p<=0.01$
D

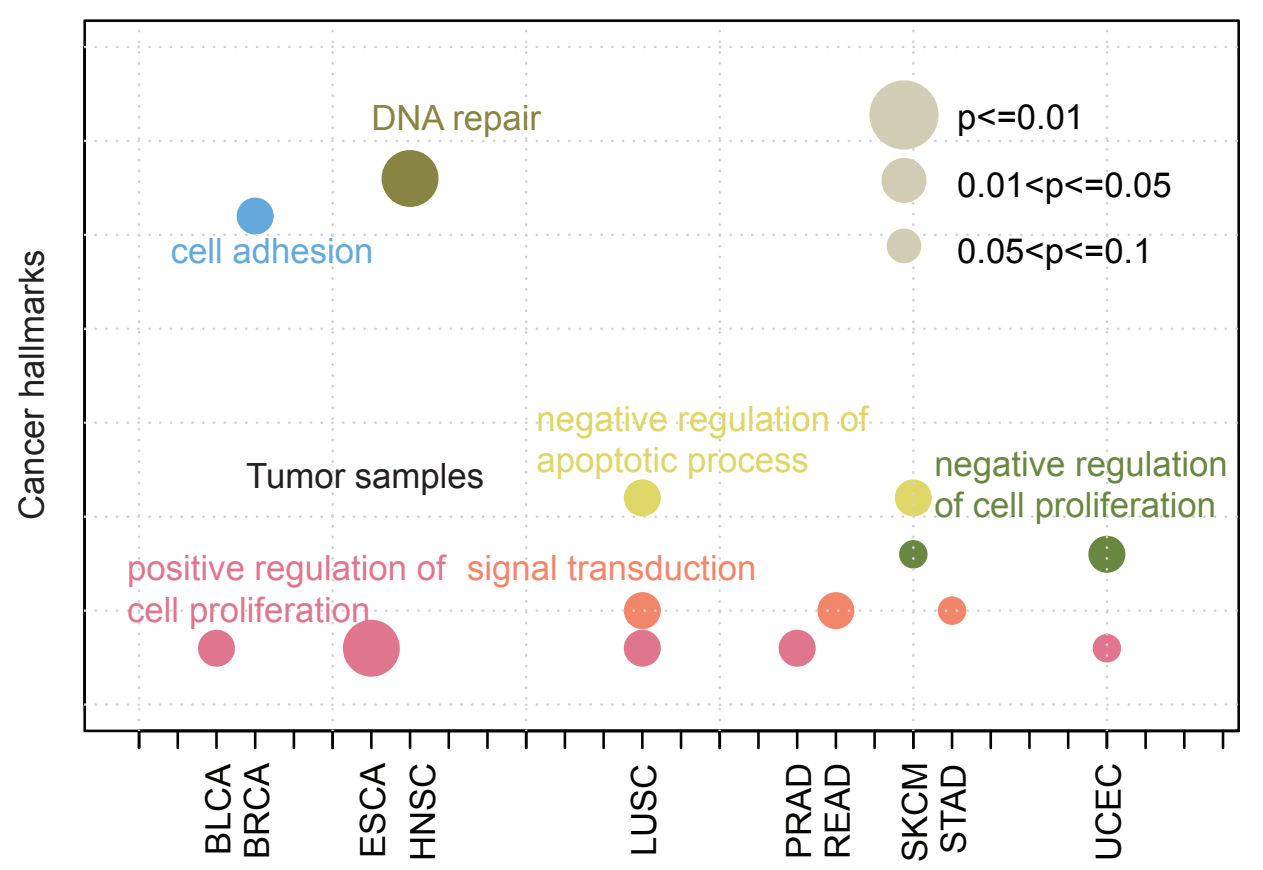


Figure 4

A
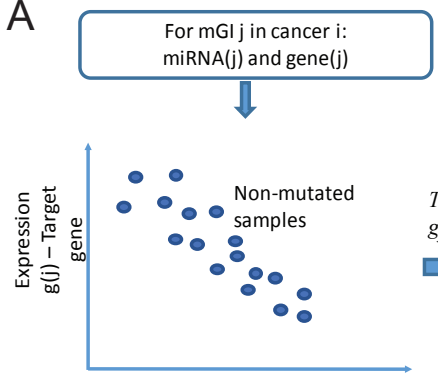

Expression $m(j)$ - miRNA

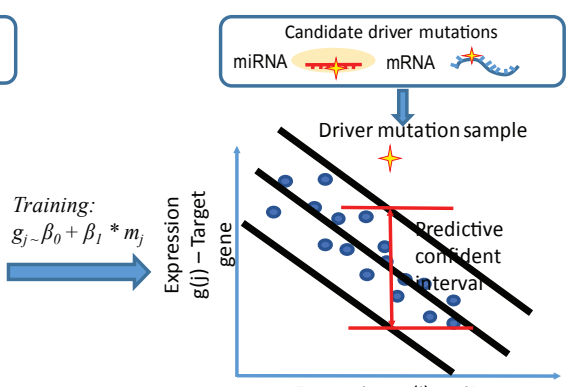

Expression $\mathrm{m}(\mathrm{j})$ - miRNA

178 driver mutations on either miRNAs or target genes

B Driver mutations across 14 cancer types (total=89)

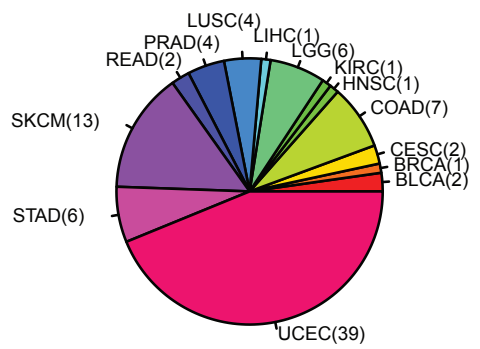

Perturbed mGls across 14 cancer types (total=154)

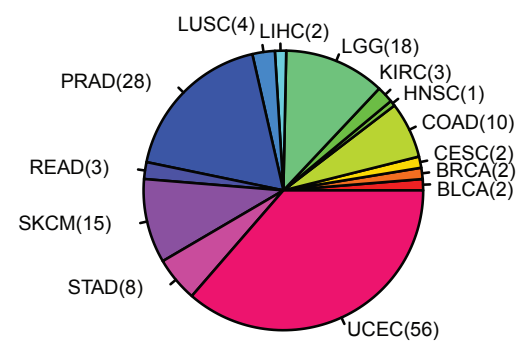

C $p=0.002 ; O R=3.3$

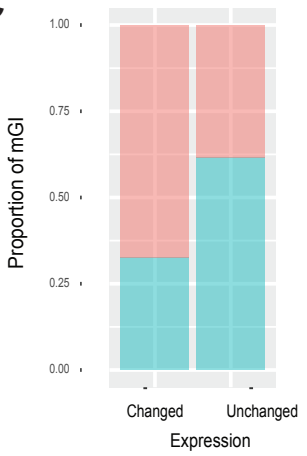

Energy changed Energy unchanged

D

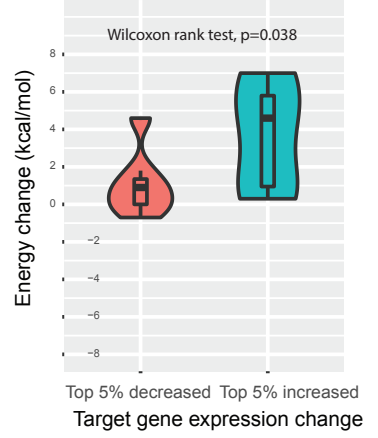


A

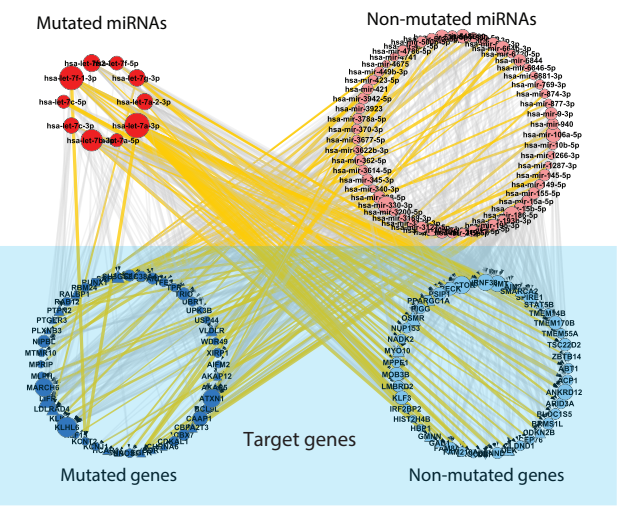

C

(40/48 mutated driver genes)

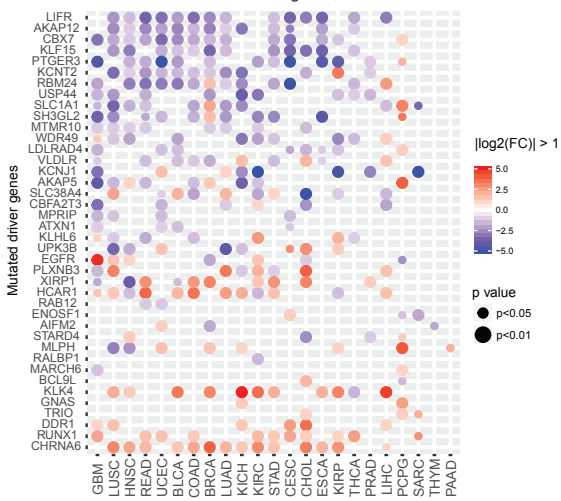

B
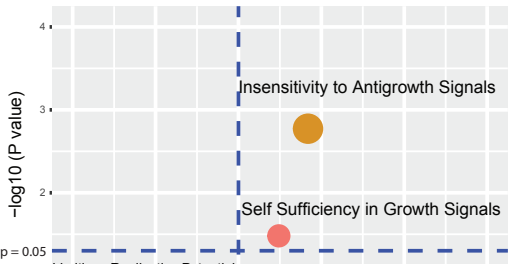

1. Limitless Replicative Potential Reprogramming Energy Metabolisn Tumor Promoting Inflammation Sustained Angiogenesis Evading Immune Detection Tissue Invasion and Metastasis Evading Apoptosis

Genome Instability and Mutalion

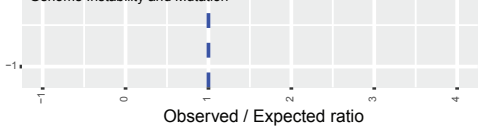

D

(31 / 45 non-mutated driver genes)

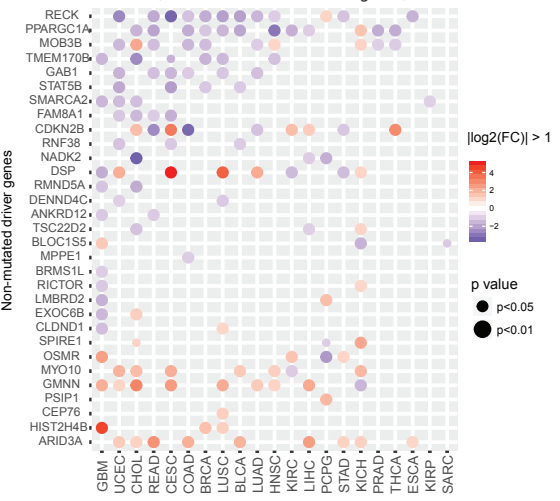


Figure 6

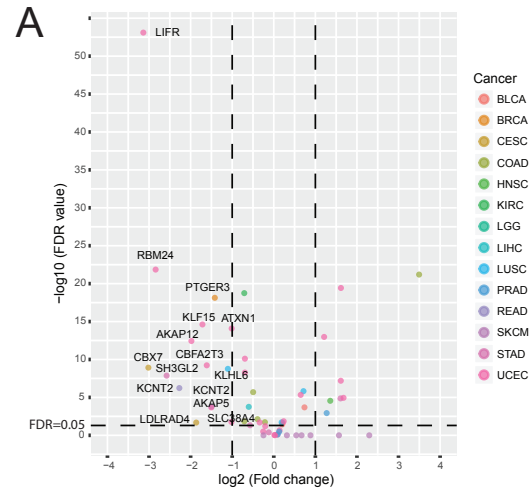

C

\begin{tabular}{|c|c|c|c|c|}
\hline Symbol & Cancer & Cancer relationship & Literature annotation & PMID \\
\hline LIFR & UCEC & $\begin{array}{l}\text { Tumor suppressor } \\
\text { Self Sufficiency in Growth } \\
\text { Signals }\end{array}$ & $\begin{array}{l}\text { LIFR confers a dormancy phenotype in } \\
\text { breast cancer cells; } \\
\text { LIFR negatively regulates the metastasis of } \\
\text { pancreatic cancer cells }\end{array}$ & $\begin{array}{l}27642788 \\
27375070\end{array}$ \\
\hline RBM24 & UCEC & Tumor suppressor & $\begin{array}{l}\text { RBM24 suppresses cancer progression in } \\
\text { nasopharyngeal carcinoma }\end{array}$ & $\begin{array}{l}27584791 \\
24356969\end{array}$ \\
\hline PTGER3 & BRCA & $\begin{array}{l}\text { Tumor suppressor } \\
\text { Self Sufficiency in Growth } \\
\text { Signals, Insensitivity to } \\
\text { Antigrowth Signals }\end{array}$ & $\begin{array}{l}\text { PTGER3 transcript levels were significantly } \\
\text { decreased in human prostate cancer }\end{array}$ & 23493387 \\
\hline KLF15 & UCEC & Tumor suppressor & $\begin{array}{l}\text { KLF15 Inhibits Cell Proliferation in Gastric } \\
\text { Cancer }\end{array}$ & 28421457 \\
\hline AKAP12 & UCEC & $\begin{array}{l}\text { Tumor suppressor } \\
\text { Self Sufficiency in Growth } \\
\text { Signals, Insensitivity to } \\
\text { Antigrowth Signals }\end{array}$ & $\begin{array}{l}\text { Tumor suppressor in GBM; Tumor } \\
\text { suppressor in prostate cancer; Tumor } \\
\text { suppressor by cell cycle }\end{array}$ & $\begin{array}{l}27593936, \\
26853466, \\
24492704 \\
21099353\end{array}$ \\
\hline $\mathrm{CBX7}$ & CESC & Tumor suppressor & $\begin{array}{l}\text { CBX7 inhibited the growth of GBM cells and } \\
\text { targeted by miR-18a; tumor suppressor in } \\
\text { pancreatic cancer. }\end{array}$ & $\begin{array}{l}28259135 \\
28123848 \\
28030829\end{array}$ \\
\hline ATXN1 & UCEC & Tumor suppressor & transcriptional repression in Notch signaling. & 21475249 \\
\hline CBFA2T3 & UCEC & $\begin{array}{l}\text { Tumor suppressor } \\
\text { Insensitivity to Antigrowth } \\
\text { Signals }\end{array}$ & $\begin{array}{l}\text { CBFA2T3 is a putative breast tumor } \\
\text { suppressor }\end{array}$ & 12183414 \\
\hline SH3GL2 & STAD & $\begin{array}{l}\text { Tumor supprressor } \\
\text { Self Sufficiency in Growth } \\
\text { Signals, Insensitivity to } \\
\text { Antigrowth Signals }\end{array}$ & $\begin{array}{l}\text { SH3GL2 gene, a novel tumor suppressor } \\
\text { gene in laryngeal squamous cell carcinoma } \\
\text { (LSCC), induces apoptosis of tumor cells }\end{array}$ & $\begin{array}{l}20512084 \\
22968441\end{array}$ \\
\hline
\end{tabular}

B

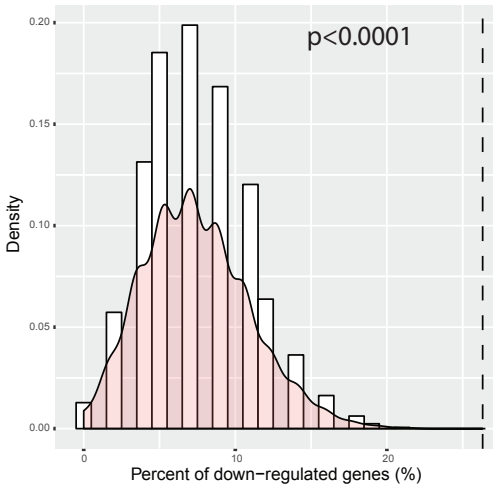

D

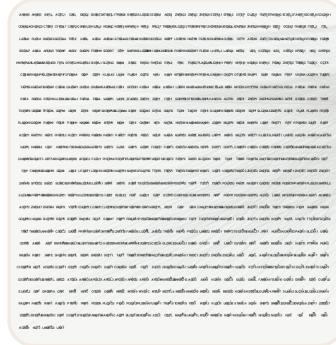

Unperturbed genes

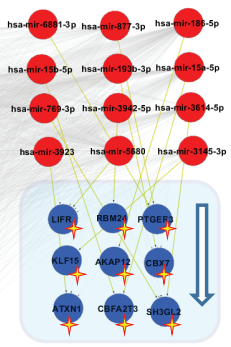

Mutated driver genes Tumor suppressors 